\title{
Prevalence and clinical significance of mediator complex subunit 12 mutations in 362 Han Chinese samples with uterine leiomyoma
}

\author{
JUAN WU ${ }^{1,2 *}$, YANG ZOU ${ }^{2,3 *}$, YONG LUO ${ }^{2,3}$, JIU-BAI GUO ${ }^{2,4}$, FA-YING LIU ${ }^{2,3}$, \\ JIANG-YAN ZHOU ${ }^{2,4}$, ZI-YU ZHANG ${ }^{2}$, LEI WAN ${ }^{2}$ and OU-PING HUANG ${ }^{1,2,4}$ \\ ${ }^{1}$ The College of Medicine, Nanchang University, Nanchang, Jiangxi 330006; \\ ${ }^{2}$ Key Laboratory of Women's Reproductive Health of Jiangxi; ${ }^{3}$ Central Laboratory and \\ ${ }^{4}$ Department of Gynecology, Jiangxi Provincial Maternal and Child Health Hospital, Nanchang, Jiangxi 330006, P.R. China
}

Received February 20, 2016; Accepted February 3, 2017

DOI: $10.3892 / \mathrm{ol} .2017 .6120$

\begin{abstract}
Uterine leiomyomas (ULs) are the most common gynecological benign tumors originating from the myometrium. Prevalent mutations in the mediator complex subunit 12 (MED12) gene have been identified in ULs, and functional evidence has revealed that these mutations may promote the development of ULs. However, whether MED12 mutations are associated with certain clinical characteristics in ULs remains largely unknown. In the present study, the potential mutations of MED12 and its paralogous gene, mediator complex subunit 12-like (MED12L), were screened in 362 UL tumors from Han Chinese patients. A total of 158 out of 362 UL tumors (43.6\%) were identified as harboring MED12 somatic mutations, and the majority of these mutations were restricted to the 44th residue. MED12 mutations were also observed in 2 out of $145(1.4 \%)$ adjacent control myometrium. Furthermore, the mutation spectrum of MED12 in the concurrent leiomyomas was noticeably different. Correlation analysis of MED12 mutations with the available clinical features indicated that patients with mutated MED12 tended to have smaller cervical diameters. By contrast, no MED12L mutation was identified in the present samples. In summary, the present study demonstrated the presence of prevalent MED12 somatic mutations in UL samples, and the MED12 mutation was associated with smaller cervical diameters. The low mutation frequency of MED12 in adjacent control myometrium indicated that MED12 mutation may be an early event in the pathogenesis of ULs.
\end{abstract}

Correspondence to: Professor Ou-Ping Huang, Key Laboratory of Women's Reproductive Health of Jiangxi, Jiangxi Provincial Maternal and Child Health Hospital, 318 Bayi Avenue, Nanchang, Jiangxi 330006, P.R. China

E-mail: jxfbhop@126.com

*Contributed equally

Key words: uterine leiomyoma, mediator complex subunit 12, mediator complex subunit 12 like, mutation, clinical characteristics
Furthermore, MED12 mutation status in concurrent tumors from multiple leiomyomas supported several prior observations that the majority of these tumors arose independently.

\section{Introduction}

Uterine leiomyomas (ULs) are the most common gynecological benign tumors originating from the myometrium, affecting $60-80 \%$ of women of reproductive age (1), and resulting in abnormal uterine bleeding, recurrent miscarriage, pelvic pain, premature birth and infertility in 10-30\% of cases (2-4). Clinically, ULs may grow as a single nodule or multiple concurrent lesions. At present, the most effective treatment strategy for ULs is a surgical operation, although certain medical drugs are also available, including gonadotropin-releasing hormone agonists and selective modulators of estrogen- and progesterone-receptors $(5,6)$.

Heterozygous somatic mutations in exon 2 of the mediator complex subunit 12 (MED12) gene located on the X chromosome have been identified in $70.7 \%(159 / 225)$ of tumors with ULs (7). Subsequent studies using the same methods or direct Sanger sequencing have also identified prevalent MED12 mutations in ULs $(8-12)$, breast fibroadenoma $(13,14)$ and chronic lymphocytic leukemia (15), whereas MED12 mutations were less common in prostate $(16,17)$ and colorectal cancer $(10,18)$. Almost all identified MED12 mutations in ULs were heterozygous somatic mutations restricted to the 44th codon (p.G44) on exon 2, only occurring at low frequencies in adjacent codons, including codons $33,36,41$ and $43(7-9,11,12)$. MED12 belongs to the mediator complex and modulates RNA polymerase II activity, and thus is involved in global gene transcriptional regulation within cells $(7,19)$. A previous in vivo study revealed that the MED12 p.G44D (c.131G>A) mutation (the most frequently observed mutation in ULs) may cause ULs and uterine hyperplasia in mice (20). Additional array comparative genomic hybridization analysis revealed that this mutation markedly increased genomic copy number gains and losses at the whole-genome scale, spanning multiple genes involved in cell cycle checkpoints and tumorigenesis (20). 
Accumulating evidence has confirmed frequent MED12 mutations in ULs (7-12). However, several issues regarding MED12 mutations in ULs remain unresolved. First, the clinical impacts of MED12 mutations in ULs remain scarce; only one study has reported that patients with MED12-mutated with ULs tend to harbor smaller sized leiomyomas compared with their counterparts without MED12 mutations (12). Second, it is largely unclear whether MED12 mutations also exist in the adjacent myometrium in patients with ULs. Third, the mutational status of MED12 in multiple concurrent leiomyomas within the same affected uterus remains unexplored in Han Chinese patients with ULs. Fourth, increasing evidence has suggested that paralogous genes are frequently mutated in certain cancer types (21-23), however, it is unknown whether MED12L (the paralogous gene of MED12) is also mutated in ULs.

To clarify these issues, MED12 and MED12L mutations were analyzed in 362 Han Chinese samples with ULs, and the results were compared with their clinical features. A high frequency $(158 / 362,43.6 \%)$ of MED12 mutations were identified in the samples used, and MED12 mutations were demonstrated to be associated with smaller cervical diameters. In addition, MED12 mutations occurred in the adjacent myometrium at a low frequency, and the mutational status of MED12 in concurrent leiomyomas from the same patients exhibited a noticeable difference. By contrast, no MED12L mutations were identified in the present sample set.

\section{Materials and methods}

Samples and clinical characteristics. A collection of 322 patients with ULs undergoing laparoscopic intracapsular myomectomy, including 362 tumors, 322 adjacent control myometrium and 322 paired peripheral blood lymphocytes, were recruited in the Department of Gynecology, Jiangxi Provincial Maternal and Child Health Hospital (Jiangxi, China) between March 2014 and February 2015. Among these patients, 290 patients had solitary leiomyomas and 32 patients had multiple leiomyomas (72 concurrent leiomyomas). All samples were diagnosed according to gynecological and abdominal ultrasonography examinations, and each patient provided informed consent. The present study conformed to the tenets of the Declaration of Helsinki and was approved by the institutional review board of Jiangxi Provincial Maternal and Child Health Hospital. Clinical characteristics of the patients are summarized in Tables I-III.

DNA isolation. Tissue and peripheral blood DNA was isolated with E.Z.N.A. tissue (cat. no. D3396-02) and blood DNA kits (cat. no. D3392-02; Omega Bio-Tek, Inc., Norcross, GA, USA), respectively. The quantity of isolated DNA was assessed using a SmartSpec Plus spectrophotometer (Bio-Rad Laboratories, Inc., Hercules, CA, USA).

MED12 and MED12L mutational analysis. For the mutational analysis of MED12 (NM_005120.2) and the paralogous MED12L (NM_053002.5; Fig. 1), two pairs of primers were designed to amplify MED12 exons 1 and 2, and MED12L exon 2. The MED12 forward (5'-TTCTCCTGCCCTACT CTCCC-3') and reverse (5'-GGACCTGGATGGACATTG

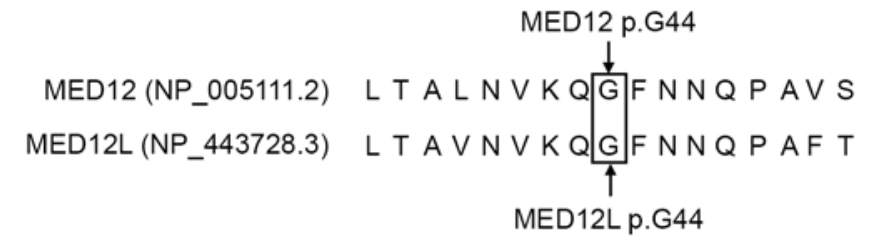

Figure 1. Protein sequence homology analysis of MED12 and MED12L. MED12, mediator complex subunit 12; MED12L, mediator complex subunit 12 like.

CA-3') primers generated a 721 bp polymerase chain reaction (PCR) product; while the MED12L forward (5'-TGA AGCTTTTACATCCTTCTGCT-3') and reverse (5'-GGG CAGGACGGTATACATGG-3') primers generated a 296 bp PCR product. For each sample, 50 ng genomic DNA and $2 \mathrm{U}$ LA Taq DNA polymerase (Takara Biotechnology Co., Ltd., Dalian, China) underwent 35 cycles of denaturation at $94^{\circ} \mathrm{C}$ for $30 \mathrm{sec}$, annealing at $60^{\circ} \mathrm{C}$ for $30 \mathrm{sec}$ and extension at $72^{\circ} \mathrm{C}$ for $20 \mathrm{sec}$, in a total volume of $25 \mu \mathrm{l}$. These reactions were performed in a Thermal Cycler 2720 (Applied Biosystems; Thermo Fisher Scientific, Inc., Waltham, MA, USA). The PCR products were subsequently sequenced on an ABI Prism 3730 DNA sequencer (Applied Biosystems; Thermo Fisher Scientific, Inc.). Sequencing data was analyzed using DNASTAR Lasergene version 7.2 software (DNASTAR, Inc., Madison, WI, USA). The identified mutations were confirmed by bidirectional sequencing, and the somatic mutations of MED12 were verified by sequencing the paired peripheral blood lymphocytes. In addition, the protein sequences of human MED12 (NP_005111.2) and MED12L (NP_443728.3) were obtained from GenBank (24) to analyze the sequence homology.

Statistical analysis. Clinical data was taken at the time of sampling for the screening of molecular alterations. As the majority of patients with multiple leiomyomas harbored wild type and mutant MED12 in different tumors, the samples with multiple leiomyomas were excluded and the available clinical data from 135 patients with single intramural leiomyoma was obtained for statistical analysis. The serum levels of estradiol, progesterone, thyroid stimulating hormone, free triiodothyronine, free thyroxine, $\alpha$-fetoprotein, carcinoembryonic antigen, squamous cell carcinoma antigen and cancer antigen 125 (CA125) were determined on day 3 of the menstrual cycle using a radioimmunoassay method, using their respective detection kits: estradiol (cat. no. 01010306024), progesterone (cat. no. 01010305024), thyroid stimulating hormone (cat. no. 01010201022), free triiodothyronine (cat. no. 01010202024), free thyroxine (cat. no. 01010203024), $\alpha$-fetoprotein (cat. no. 01010102022), carcinoembryonic antigen (cat. no. 01010101022 ), and CA125 (cat. no. 01010104022) from Chemclin (Chemclin, Biotechnology Co., Ltd., Beijing, China), and squamous cell carcinoma antigen (cat. no. 68111LP15) was from Abbott Diagnostics (Santa Clara, CA, USA), as described previously (25-27). Statistical analyses were performed with SPSS software, version 17.0 (SPSS, Inc., Chicago, IL, USA). Continuous variables were expressed as the mean \pm standard deviation 
Table I. Association of mediator complex subunit 12 mutations with clinical characteristics in 135 uterine leiomyomas.

\begin{tabular}{|c|c|c|c|c|c|c|}
\hline Features & Sample, $\mathrm{n}$ & Wild type, $n$ & Wild type & Mutant type, $\mathrm{n}$ & Mutant type & P-value \\
\hline Total & 135 & 86 & & 49 & & \\
\hline Age (years) & 135 & 86 & $41.51 \pm 6.52$ & 49 & $39.61 \pm 7.09$ & 0.12 \\
\hline Duration of disease (months) & 135 & 86 & $30.05 \pm 41.65$ & 49 & $32.19 \pm 39.46$ & 0.78 \\
\hline Age of menarche (years) & 129 & 82 & $14.61 \pm 1.64$ & 47 & $14.11 \pm 1.46$ & 0.088 \\
\hline Age of the first delivery (years) & 117 & 76 & $23.07 \pm 2.62$ & 41 & $22.88 \pm 2.63$ & 0.718 \\
\hline Number of pregnancies (times) & 135 & 86 & $3.05 \pm 1.49$ & 49 & $3.06 \pm 1.64$ & 0.96 \\
\hline Number of deliveries (times) & 135 & 86 & $1.67 \pm 0.89$ & 49 & $1.63 \pm 0.95$ & 0.85 \\
\hline Number of abortions (times) & 135 & 86 & $1.37 \pm 1.49$ & 49 & $1.39 \pm 1.27$ & 0.95 \\
\hline Cervical diameter $(\mathrm{cm})$ & 132 & 84 & $3.15 \pm 0.32$ & 48 & $3.04 \pm 0.22$ & 0.030 \\
\hline Haemoglobin $(\mathrm{g} / \mathrm{l})$ & 135 & 86 & $109.09 \pm 21.60$ & 49 & $111.53 \pm 21.10$ & 0.53 \\
\hline Estrogen level (pg/ml) & 74 & 44 & $157.02 \pm 137.44$ & 30 & $135.89 \pm 111.65$ & 0.49 \\
\hline Progesterone (ng/ml) & 66 & 39 & $1.48 \pm 3.31$ & 27 & $0.76 \pm 0.86$ & 0.27 \\
\hline TSH (mIU/ml) & 128 & 83 & $2.99 \pm 2.04$ & 45 & $3.19 \pm 4.43$ & 0.73 \\
\hline FT3 (pg/ml) & 128 & 83 & $2.89 \pm 0.35$ & 45 & $2.86 \pm 0.38$ & 0.70 \\
\hline FT4 (ng/dl) & 128 & 83 & $1.20 \pm 0.19$ & 45 & $1.20 \pm 0.13$ & 0.84 \\
\hline AFP (ng/ml) & 130 & 83 & $2.28 \pm 1.43$ & 47 & $2.00 \pm 0.79$ & 0.23 \\
\hline CEA (ng/ml) & 130 & 83 & $1.21 \pm 0.631$ & 47 & $1.18 \pm 0.64$ & 0.79 \\
\hline CA125 $(\mu / \mathrm{ml})$ & 131 & 84 & $17.97 \pm 11.06$ & 47 & $27.21 \pm 58.83$ & 0.16 \\
\hline $\mathrm{SCC}(\mathrm{ng} / \mathrm{ml})$ & 129 & 82 & $1.74 \pm 1.52$ & 47 & $1.65 \pm 1.25$ & 0.73 \\
\hline HPV infection (pg/ml) & 92 & 61 & $3.61 \pm 21.38$ & 31 & $4.14 \pm 13.73$ & 0.90 \\
\hline White blood cell count $\left(\mathrm{x} 10^{9}\right)$ & 135 & 86 & $5.95 \pm 2.02$ & 49 & $5.64 \pm 1.65$ & 0.37 \\
\hline Lymphocyte cell count $\left(\times 10^{9}\right)$ & 135 & 86 & $1.73 \pm 0.51$ & 49 & $1.70 \pm 0.41$ & 0.75 \\
\hline Mononuclear cell count $\left(\mathrm{x} 10^{9}\right)$ & 135 & 86 & $0.48 \pm 0.18$ & 49 & $0.43 \pm 0.14$ & 0.15 \\
\hline Neutrophil count $\left(\mathrm{x} 10^{9}\right)$ & 135 & 86 & $0.10 \pm 0.07$ & 49 & $0.17 \pm 0.21$ & 0.0023 \\
\hline
\end{tabular}

AFP, $\alpha$-fetoprotein; TSH, thyroid stimulating hormone; FT3, free triiodothyronine; FT4, free thyroxine; CEA, carcinoembryonic antigen; CA125, cancer antigen 125; SCC, squamous cell carcinoma; HPV, human papillomavirus.

Table II. Association between mediator complex subunit 12 mutations and clinical characteristics in 135 uterine leiomyomas.

\begin{tabular}{|c|c|c|c|c|c|}
\hline \multirow[b]{2}{*}{ Features } & \multicolumn{2}{|c|}{ Wild type $(n=86)$} & \multicolumn{2}{|c|}{ Mutant type $(n=49)$} & \multirow[b]{2}{*}{ P-value } \\
\hline & Regular, $\mathrm{n}$ & Irregular, $\mathrm{n}$ & Regular, $\mathrm{n}$ & Irregular, $\mathrm{n}$ & \\
\hline Menstruation & 79 & 7 & 48 & 1 & 0.26 \\
\hline Menstrual cycle & 78 & 8 & 45 & 4 & 1.00 \\
\hline Caesarean birth & & & & & 0.32 \\
\hline Yes & 9 & & 8 & & \\
\hline No & & 77 & & 41 & \\
\hline Cervical erosion & 48 & 38 & 31 & 18 & 0.93 \\
\hline
\end{tabular}

and analyzed by independent sample t-tests, and categorical variables were examined with Pearson's $\chi^{2}$ test for the potential associations between MED12 mutations and the available biological variables. Clinical characteristics of interest were analyzed by logistic regression analysis to confirm statistical significance. All statistical tests were two-sided at the $5 \%$ level of significance. $\mathrm{P}<0.05$ was considered to indicate a statistically significant difference, and all P-values were two-tailed.

\section{Results}

Patient characteristics. Patient characteristics are presented in Tables I-III.

Mutation analysis of MED12 and MED12L in ULs. A total of 362 cases with ULs were analyzed for the presence of MED12 and MED12L mutations. MED12 mutations were identified in 158 out of 362 tumors $(43.6 \%)$. There were 10 types of 
Table III. Association between mediator complex subunit 12 mutations and clinical characteristics in 135 uterine leiomyomas.

\begin{tabular}{|c|c|c|c|c|c|c|c|}
\hline \multirow[b]{2}{*}{ Feature } & \multicolumn{3}{|c|}{ Wild type, $\mathrm{n}$} & \multicolumn{3}{|c|}{ Mutant type, n } & \multirow[b]{2}{*}{ P-value } \\
\hline & Less & Median & More & Less & Median & More & \\
\hline Menstrual blood & & & & & & & 0.51 \\
\hline None & 5 & & & 1 & & & \\
\hline Less & & 65 & & & 40 & & \\
\hline Common & & & 15 & & & 1 & \\
\hline Dysmenorrhea & 72 & 6 & 8 & 35 & 12 & 16 & 0.24 \\
\hline
\end{tabular}

Table IV. Mutation frequencies and forms of mediator complex subunit 12 in tumors with uterine leiomyomas.

\begin{tabular}{|c|c|c|c|}
\hline Disease & Nucleotide change & Protein change & No. mutated samples, frequency $(\%)$ \\
\hline \multicolumn{4}{|l|}{ Solitary } \\
\hline & c. $107 \mathrm{G}>\mathrm{T}$ & p.L36R & $5 / 290(1.7)$ \\
\hline & c. $122 \mathrm{~T}>\mathrm{A}$ & p.V41E & $1 / 290(0.3)$ \\
\hline & c. $128 \mathrm{~A}>\mathrm{C}$ & p.Q43P & $1 / 290(0.3)$ \\
\hline & c. $130 \mathrm{G}>\mathrm{A}$ & p.G44S & $14 / 290(4.8)$ \\
\hline & c. $130 \mathrm{G}>\mathrm{T}$ & p.G44C & $9 / 290(3.1)$ \\
\hline & c. $130 \mathrm{G}>\mathrm{C}$ & p.G44R & $5 / 290(1.7)$ \\
\hline & c. $131 \mathrm{G}>\mathrm{A}$ & p.G44D & $53 / 290(18.3)$ \\
\hline & c. $131 \mathrm{G}>\mathrm{T}$ & p.G44V & $22 / 290(7.6)$ \\
\hline & c. $131 \mathrm{G}>\mathrm{C}$ & p.G44A & $8 / 290(2.8)$ \\
\hline & c.133_150del 18 & p.F45_A50del & $3 / 290(0.7)$ \\
\hline \multirow[t]{8}{*}{ Total multiple } & & & $121 / 290(42.4)$ \\
\hline & c. $107 \mathrm{G}>\mathrm{T}$ & p.L36R & $4 / 72(5.6)$ \\
\hline & c. $130 \mathrm{G}>\mathrm{A}$ & p.G44V & $2 / 72(2.8)$ \\
\hline & c. $130 \mathrm{G}>\mathrm{T}$ & p.G44C & $4 / 72(5.6)$ \\
\hline & c. $130 \mathrm{G}>\mathrm{C}$ & p.G44R & 4/72 (5.6) \\
\hline & c. $131 \mathrm{G}>\mathrm{A}$ & p.G44D & 14/72 (19.4) \\
\hline & c. $131 \mathrm{G}>\mathrm{T}$ & p.G44S & $2 / 72(2.8)$ \\
\hline & c. $131 \mathrm{G}>\mathrm{C}$ & p.G44A & 7/72 (9.7) \\
\hline \multirow[t]{11}{*}{ Total combined } & & & $37 / 72(51.4)$ \\
\hline & c. $107 \mathrm{G}>\mathrm{T}$ & p.L36R & $9 / 362(2.5)$ \\
\hline & c. $122 \mathrm{~T}>\mathrm{A}$ & p.V41E & $1 / 362(0.3)$ \\
\hline & c. $128 \mathrm{~A}>\mathrm{C}$ & p.Q43P & $1 / 362(0.3)$ \\
\hline & c. $130 \mathrm{G}>\mathrm{A}$ & p.G44S & $16 / 362(4.4)$ \\
\hline & c. $130 \mathrm{G}>\mathrm{T}$ & p.G44C & $13 / 362(3.6)$ \\
\hline & c. $130 \mathrm{G}>\mathrm{C}$ & p.G44R & $9 / 362(2.5)$ \\
\hline & c. $131 \mathrm{G}>\mathrm{A}$ & p.G44D & 67/362 (18.5) \\
\hline & c. $131 \mathrm{G}>\mathrm{T}$ & p.G44V & $24 / 362(6.6)$ \\
\hline & c. $131 \mathrm{G}>\mathrm{C}$ & p.G44A & $15 / 362(4.1)$ \\
\hline & c.133_150del 18 & p.F45_A50del & 3/362 (0.8) \\
\hline Total & & & $158 / 362(43.6)$ \\
\hline
\end{tabular}

mutation, including p.L36R (c.107T $>$ G), p.V41E (c.122T $>$ A), p.Q43P (c.128A>C), p.G44S (c.130G>A), p.G44C (c.130G>T), p.G44R (c.130G>C), p.G44D (c.131G>A), p.G44 V (c.131G>T), p.G44A (c.131G>C) and p.F45_A50del (c.133_150del 18; Fig. 2; Table IV). Of these mutations, the most prevalent mutation was located on the 44th codon, and MED12 p.G44D (c.131G $>$ A) was the most common mutation form $(42.4 \%$, 67/158). All patients with the mutation were heterozygous and retained a wild type allele, and the somatic status of this sample was confirmed by sequencing the paired peripheral 
Table V. MED12 mutation analysis in concurrent tumors from patients with multiple leiomyomas.

\begin{tabular}{|c|c|c|}
\hline Sample no. & Tumor no. & MED12 status \\
\hline 198 & 198T1 & WT \\
\hline 198 & 198T2 & c.107T>G, p.L36R \\
\hline 200 & $200 \mathrm{~T} 1$ & c. $131 \mathrm{G}>\mathrm{A}, \mathrm{p} . \mathrm{G} 44 \mathrm{D}$ \\
\hline 200 & $200 \mathrm{~T} 2$ & WT \\
\hline 224 & 224T1 & c. $131 \mathrm{G}>\mathrm{T}, \mathrm{p} . \mathrm{G} 44 \mathrm{~V}$ \\
\hline 224 & $224 \mathrm{~T} 2$ & c.130G >C, p.G44R \\
\hline 366 & $366 \mathrm{~T} 1$ & c.130G >C, p.G44R \\
\hline 366 & $366 \mathrm{~T} 2$ & c. $131 \mathrm{G}>\mathrm{A}, \mathrm{p} . \mathrm{G} 44 \mathrm{D}$ \\
\hline 388 & 388T1 & c. $131 \mathrm{G}>\mathrm{A}, \mathrm{p} . \mathrm{G} 44 \mathrm{D}$ \\
\hline 388 & 388T2 & c.107T>G, p.L36R \\
\hline 391 & $391 \mathrm{~T} 1$ & WT \\
\hline 391 & $391 \mathrm{~T} 2$ & WT \\
\hline 391 & $391 \mathrm{~T} 3$ & WT \\
\hline 411 & $411 \mathrm{~T} 1$ & WT \\
\hline 411 & $411 \mathrm{~T} 2$ & WT \\
\hline 411 & $411 \mathrm{~T} 3$ & WT \\
\hline 415 & $415 \mathrm{~T} 1$ & c. $130 \mathrm{G}>\mathrm{T}, \mathrm{p} . \mathrm{G} 44 \mathrm{C}$ \\
\hline 415 & $415 \mathrm{~T} 2$ & c. $130 \mathrm{G}>\mathrm{C}, \mathrm{p} . \mathrm{G} 44 \mathrm{R}$ \\
\hline 415 & $415 \mathrm{~T} 3$ & WT \\
\hline 416 & 416T1 & c. $131 \mathrm{G}>\mathrm{A}, \mathrm{p} . \mathrm{G} 44 \mathrm{D}$ \\
\hline 416 & $416 \mathrm{~T} 2$ & c.131G >T, p.G44V \\
\hline 420 & $420 \mathrm{~T} 1$ & c. $131 \mathrm{G}>\mathrm{A}, \mathrm{p} . \mathrm{G} 44 \mathrm{D}$ \\
\hline 420 & $420 \mathrm{~T} 2$ & c. $131 \mathrm{G}>\mathrm{A}, \mathrm{p} . \mathrm{G} 44 \mathrm{D}$ \\
\hline 420 & $420 \mathrm{~T} 3$ & c. $131 \mathrm{G}>\mathrm{A}, \mathrm{p} . \mathrm{G} 44 \mathrm{D}$ \\
\hline 422 & $422 \mathrm{~T} 1$ & c.130G >T, p.G44C \\
\hline 422 & $422 \mathrm{~T} 2$ & c. $131 \mathrm{G}>\mathrm{C}, \mathrm{p} . \mathrm{G} 44 \mathrm{~A}$ \\
\hline 423 & $423 \mathrm{~T} 1$ & c. $130 \mathrm{G}>\mathrm{T}, \mathrm{p} . \mathrm{G} 44 \mathrm{C}$ \\
\hline 423 & $423 \mathrm{~T} 2$ & WT \\
\hline 424 & 424T1 & c. $130 \mathrm{G}>\mathrm{T}, \mathrm{p} . \mathrm{G} 44 \mathrm{C}$ \\
\hline 424 & $424 \mathrm{~T} 2$ & c. $131 \mathrm{G}>\mathrm{C}, \mathrm{p} . \mathrm{G} 44 \mathrm{~A}$ \\
\hline 459 & 459T1 & WT \\
\hline 459 & $459 \mathrm{~T} 2$ & c. $131 \mathrm{G}>\mathrm{C}, \mathrm{p} . \mathrm{G} 44 \mathrm{~A}$ \\
\hline 481 & $481 \mathrm{~T} 1$ & c.107T>G, p.L36R \\
\hline 481 & $481 \mathrm{~T} 2$ & WT \\
\hline 481 & $481 \mathrm{~T} 3$ & WT \\
\hline 482 & $482 \mathrm{~T} 1$ & WT \\
\hline 482 & $482 \mathrm{~T} 2$ & WT \\
\hline 488 & $488 \mathrm{~T} 1$ & c.130G >A, p.G44S \\
\hline 488 & $488 \mathrm{~T} 2$ & WT \\
\hline 489 & 489T1 & WT \\
\hline 489 & 489T2 & WT \\
\hline 491 & $491 \mathrm{~T} 1$ & c. $131 \mathrm{G}>\mathrm{A}, \mathrm{p} . \mathrm{G} 44 \mathrm{D}$ \\
\hline 491 & $491 \mathrm{~T} 2$ & c. $131 \mathrm{G}>\mathrm{C}, \mathrm{p} . \mathrm{G} 44 \mathrm{~A}$ \\
\hline 494 & 494T1 & c. $131 \mathrm{G}>\mathrm{C}, \mathrm{p} . \mathrm{G} 44 \mathrm{~A}$ \\
\hline 494 & 494T2 & c. $130 \mathrm{G}>\mathrm{A}, \mathrm{p} . \mathrm{G} 44 \mathrm{~S}$ \\
\hline 497 & 497T1 & WT \\
\hline 497 & 497T2 & WT \\
\hline 498 & 498T1 & WT \\
\hline 498 & 498T2 & WT \\
\hline 499 & 499T1 & c. $131 \mathrm{G}>\mathrm{A}, \mathrm{p} . \mathrm{G} 44 \mathrm{D}$ \\
\hline 499 & 499T2 & WT \\
\hline
\end{tabular}

Table V. Continued.

\begin{tabular}{lcc}
\hline Sample no. & Tumor no. & MED12 status \\
\hline 500 & $500 \mathrm{~T} 1$ & c.131G $>$ A, p.G44D \\
500 & $500 \mathrm{~T} 2$ & c.131G $>$ A, p.G44D \\
501 & $501 \mathrm{~T} 1$ & WT \\
501 & $501 \mathrm{~T} 2$ & WT \\
504 & $504 \mathrm{~T} 1$ & WT \\
504 & $504 \mathrm{~T} 2$ & WT \\
506 & $506 \mathrm{~T} 1$ & WT \\
506 & $506 \mathrm{~T} 2$ & WT \\
524 & $524 \mathrm{~T} 1$ & WT \\
524 & $524 \mathrm{~T} 2$ & $\mathrm{c} .131 \mathrm{G}>$ A, $\mathrm{p} . \mathrm{G} 44 \mathrm{D}$ \\
530 & $530 \mathrm{~T} 1$ & $\mathrm{c} .131 \mathrm{G}>\mathrm{A}, \mathrm{p} . \mathrm{G} 44 \mathrm{D}$ \\
530 & $530 \mathrm{~T} 2$ & $\mathrm{c} .131 \mathrm{G}>\mathrm{C}, \mathrm{p} . \mathrm{G} 44 \mathrm{~A}$ \\
530 & $530 \mathrm{~T} 3$ & $\mathrm{c} .131 \mathrm{G}>\mathrm{C}, \mathrm{p} . \mathrm{G} 44 \mathrm{~A}$ \\
531 & $531 \mathrm{~T} 1$ & $\mathrm{c} .131 \mathrm{G}>\mathrm{A}, \mathrm{p} . \mathrm{G} 44 \mathrm{D}$ \\
531 & $531 \mathrm{~T} 2$ & WT \\
531 & $531 \mathrm{~T} 3$ & WT \\
576 & $576 \mathrm{~T} 1$ & WT \\
576 & $576 \mathrm{~T} 2$ & WT \\
580 & $580 \mathrm{~T} 1$ & c.130G $>$ C, p.G44R \\
580 & $580 \mathrm{~T} 2$ & \\
580 & $580 \mathrm{~T} 3$ & \\
\hline
\end{tabular}

WT, wild type; MED12, mediator complex subunit 12 .

blood (Fig. 2). In contrast to the frequent MED12 mutations, no MED12L mutations were identified in the 362 samples with ULs (Fig. 2).

MED12 mutations in multiple ULs. To determine the frequency of clonally associated ULs in the samples, 72 concurrent tumors from 32 patients with multiple leiomyomas were analyzed for the presence of MED12 mutations. A total of 45 tumors from 20 patients exhibited discordant mutation status of MED12, indicating that these tumors were clonally independent (Table V).

MED12 mutations in adjacent control myometrium. A total of 145 adjacent control myometrium adjacent to MED12-mutated tumors were analyzed for the presence of MED12 mutations by direct DNA sequencing. Two myometrium $(2 / 145,1.4 \%)$ were revealed to harbor MED12 mutations. Control myometrium from samples 387 and 395 harbored MED12 p.G44D (c.131G >A) and p.G44S (c.130G >A) mutations, respectively (Table VI), while the remaining 143 myometrium did not harbor MED12 mutations.

Association between MED12 mutations, clinical features and biological characteristics. Of the 322 patients, MED12-mutated tumors tended to have smaller cervical diameters [odds ratio $(\mathrm{OR})=0.181 ; 95 \%$ confidence interval, 0.036-0.919; $\mathrm{P}<0.05$; Tables I and VII]. However, MED12 mutations were not associated with any other clinical characteristics, including age of menarche and the first delivery, number of pregnancies, 
A

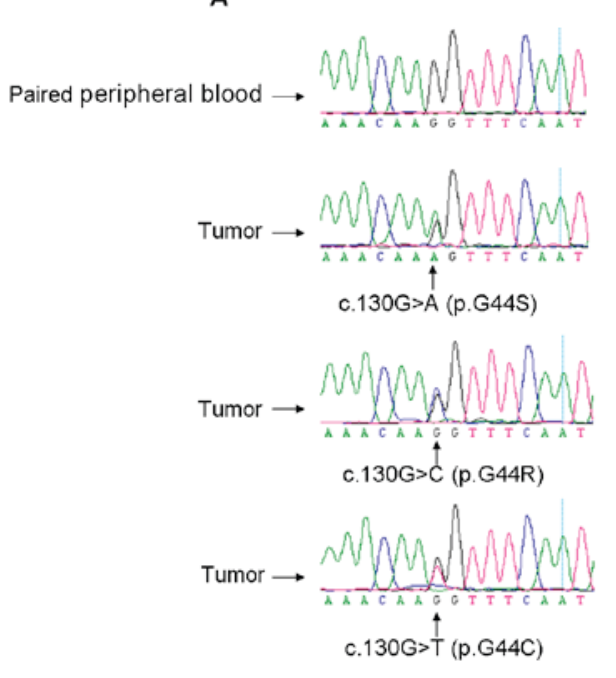

B

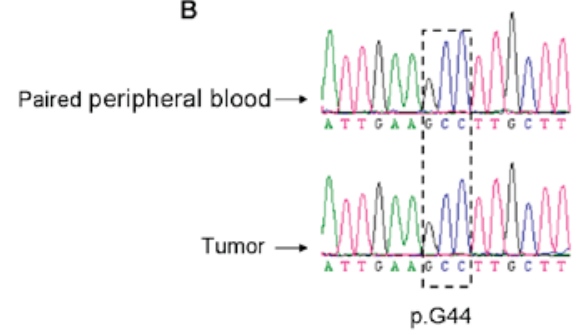

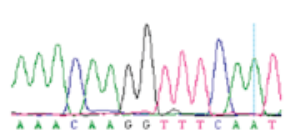
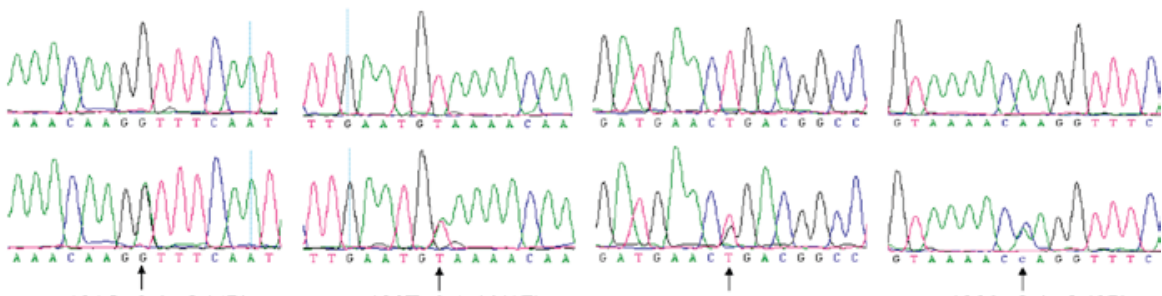

c.122T>A (p.V41E)

c. $107 T>G(p . L 36 R)$

c.128A>C (p.Q43P)

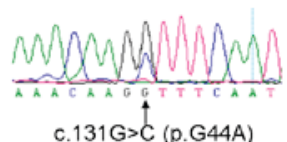

Paired peripheral blood
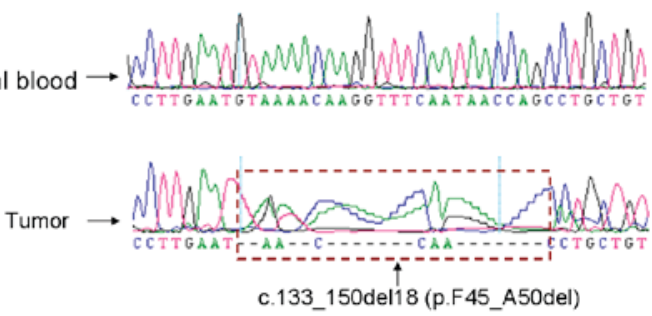

Figure 2. Representative sequencing electropherograms of (A) mediator complex subunit 12 and (B) mediator complex subunit 12-like. Arrows refer to the locations of the mutation.

Table VI. MED12 mutation analysis in adjacent control myometrium in patients with uterine leiomyomas.

\begin{tabular}{lll}
\hline $\begin{array}{l}\text { Patient } \\
\text { number }\end{array}$ & \multicolumn{1}{c}{ Tissue } & \multicolumn{1}{c}{$\begin{array}{c}\text { MED12 } \\
\text { mutation }\end{array}$} \\
\hline 387 & Leiomyoma & c.130G $>$ A, p.G44S \\
& Adjacent myometrium & c.131G $>$ A, p.G44D \\
395 & Leiomyoma & c.130G $>$ C, p.G44R \\
& Adjacent myometrium & c.130G $>$ A, p.G44S \\
\hline
\end{tabular}

MED12, mediator complex subunit 12.

deliveries and abortions, menstrual cycle and menstruation, or serum levels of estradiol, progesterone and CA125 (Tables I-III).

\section{Discussion}

Prevalent MED12 mutations have been detected in ULs (8-12), breast fibroadenoma $(13,14)$ and chronic lymphocytic leukemia (15). Evolutionary conservation analysis results have demonstrated that these mutations were evolutionarily conserved from yeast to humans (18). Furthermore, transgenic mice expressing MED12 p.G44D mutations demonstrated increased genomic instability, uterine hyperplasia and ULs (20).

Previous studies have revealed variable frequencies of MED12 mutations in ULs, ranging from $0-84.1 \%$
Table VII. Logistic regression analysis demonstrating that mediator complex subunit 12 mutations are associated with smaller cervical diameters in uterine leiomyomas.

\begin{tabular}{lcc}
\hline Feature & $\begin{array}{c}\text { Odds ratio } \\
(95 \% \text { confidence interval })\end{array}$ & P-value \\
\hline Cervical diameter & $0.181(0.036-0.919)$ & 0.039 \\
\hline
\end{tabular}

(7,8,10-12,28-37). Among which, MED12 mutation frequencies in Han Chinese UL samples were $29.3 \%$ (49/167) (35), $52.5 \%(95 / 181)(33)$ and $54.4 \%$ (93/171) (38), respectively, within the previously observed frequency. In the present study, the mutation frequency of MED12 in the UL samples was $158 / 362$ (43.6\%), within the range of these previous observations (7,8,10-12,28-37). In addition, the prevalent MED12 mutations in Han Chinese ULs observed in the present study as well as previous studies $(35,38)$ indicated that MED12 mutations may be actively involved in the pathogenesis of ULs in Han Chinese patients. It was hypothesized that the variation of MED12 mutations in ULs may be caused by disease heterogeneity, as ULs are subdivided into multiple different subtypes $(28,35,37)$.

MED12 mutations have been investigated broadly in samples with ULs $(7,8,10-12,28-37)$. However, only one study has explored the potential associations between MED12 and several clinical features, which analyzed a total of 164 ULs 
from Finnish patients and identified that MED12 mutations were associated with smaller tumor size and a larger number of tumors in multiple leiomyomas (12). Similarly, the present results also revealed that patients with MED12-mutated tumors tended to have smaller cervical diameters when compared with patients with MED12 wild type tumors. The consistent observations in this previous and present study indicated that MED12 mutations were inversely associated with cervix size in ULs. In addition, in the present study, no associations were observed between MED12 mutations and other available clinical data, including age of menarche and the first delivery, numbers of pregnancies, deliveries and abortions, menstrual cycle and menstruation, or serum levels of estradiol, progesterone and CA125 (12).

Previous studies have also attempted to determine whether MED12 mutations also existed in the adjacent control myometrium of ULs $(7,31,34,39,40)$. MED12 mutations were identified in 2 out of $13(15 \%)$ cases in myometrium adjacent to leiomyomas (39), while several other studies did not detect MED12 mutations in the control myometrium $(7,31,34,40)$. A low frequency of MED12 mutations was detected in 2 out of $145(1.4 \%)$ adjacent control myometrium with MED12 mutations in the tumors (Table IV). The exact reason for the differential MED12 mutation frequency in control myometrium was unclear, however, the presence of MED12 mutations in the adjacent myometrium in ULs indicated that MED12 mutation may be an early event in the development of ULs in certain cases.

The MED12 mutation status in concurrent tumors from multiple leiomyomas supported several previous observations that the majority of these tumors arise independently $(7,9)$.

In contrast to the high frequency of MED12 mutations in ULs, no mutations were detected in the paralogous MED12L in the present sample cohort, indicating that MED12L mutations may not be common in ULs.

In summary, the present study observed prevalent MED12 mutations in UL samples. Positive MED12 mutations were associated with the diameter of uterine cervix in ULs. Furthermore, MED12 mutations were observed at low frequencies in adjacent control myometrium in ULs, indicating that MED12 mutation may be an early event in the pathogenesis of a proportion of ULs. Furthermore, MED12 mutation status in concurrent tumors from multiple leiomyomas supported several prior observations that the majority of these tumors arise independently.

\section{Acknowledgements}

The present study was supported by the National Natural Science Foundation of China (grant nos. 81060052 and 81260097) and the GanPo Outstanding Talents 555 Project of Jiangxi Province.

\section{References}

1. Levy G, Hill MJ, Plowden TC, Catherino WH and Armstrong AY: Biomarkers in uterine leiomyoma. Fertil Steril 99: 1146-1152, 2013.

2. Bradley LD and Gueye NA: The medical management of abnormal uterine bleeding in reproductive-aged women. Am J Obstet Gynecol 214: 31-44, 2016.

3. Commandeur AE, Styer AK and Teixeira JM: Epidemiological and genetic clues for molecular mechanisms involved in uterine leiomyoma development and growth. Hum Reprod Update 21: 593-615, 2015.
4. Marino JL, Eskenazi B, Warner M, Samuels S, Vercellini P, Gavoni N and Olive D: Uterine leiomyoma and menstrual cycle characteristics in a population-based cohort study. Hum Reprod 19: 2350-2355, 2004.

5. Chabbert-Buffet N, Esber N and Bouchard P: Fibroid growth and medical options for treatment. Fertil Steril 102: 630-639, 2014.

6. Donnez J, Donnez O and Dolmans MM: With the advent of selective progesterone receptor modulators, what is the place of myoma surgery in current practice? Fertil Steril 102: 640-648, 2014.

7. Makinen N, Mehine M, Tolvanen J, Kaasinen E,Li Y,Lehtonen HJ, Gentile M, Yan J, Enge M, Taipale M, et al: MED12, the mediator complex subunit 12 gene, is mutated at high frequency in uterine leiomyomas. Science 334: 252-255, 2011.

8. Mehine M, Kaasinen E, Mäkinen N, Katainen R, Kämpjärvi K, Pitkänen E, Heinonen HR, Bützow R, Kilpivaara O, Kuosmanen A, et al: Characterization of uterine leiomyomas by whole-genome sequencing. N Engl J Med 369: 43-53, 2013.

9. Markowski DN, Bartnitzke S, Löning T, Drieschner N, Helmke BM and Bullerdiek J: MED12 mutations in uterine fibroids-their relationship to cytogenetic subgroups. Int J Cancer 131: 1528-1536, 2012.

10. Kämpjärvi K, Park MJ, Mehine M, Kim NH, Clark AD Bützow R, Böhling T, Böhm J, Mecklin JP, Järvinen H, et al: Mutations in exon 1 highlight the role of MED12 in uterine leiomyomas. Hum Mutat 35: 1136-1141, 2014.

11. Di Tommaso S, Tinelli A, Malvasi A and Massari S: Missense mutations in exon 2 of the MED12 gene are involved in IGF-2 overexpression in uterine leiomyoma. Mol Hum Reprod 20: 1009-1015, 2014.

12. Heinonen HR, Sarvilinna NS, Sjöberg J, Kämpjärvi K, Pitkänen E, Vahteristo P, Mäkinen N and Aaltonen LA: MED12 mutation frequency in unselected sporadic uterine leiomyomas. Fertil Steril 102: 1137-1142, 2014.

13. Lim WK, Ong CK, Tan J, Thike AA, Ng CC, Rajasegaran V, Myint SS, Nagarajan S, Nasir ND, McPherson JR, et al: Exome sequencing identifies highly recurrent MED12 somatic mutations in breast fibroadenoma. Nat Genet 46: 877-880, 2014.

14. Tan J, Ong CK, Lim WK, Ng CC, Thike AA, Ng LM, Rajasegaran V, Myint SS, Nagarajan S and Thangaraju S: Genomic landscapes of breast fibroepithelial tumors. Nat Genet 47: 1341-1345, 2015.

15. Kämpjärvi K, Järvinen TM, Heikkinen T, Ruppert AS, Senter L, Hoag KW, Dufva O, Kontro M, Rassenti L, Hertlein E, et al: Somatic MED12 mutations are associated with poor prognosis markers in chronic lymphocytic leukemia. Oncotarget 6: 1884-1888, 2015.

16. Barbieri CE, Baca SC, Lawrence MS, Demichelis F, Blattner M, Theurillat JP, White TA, Stojanov P, Van Allen E, Stransky N, et al: Exome sequencing identifies recurrent SPOP, FOXA1 and MED12 mutations in prostate cancer. Nat Genet 44: 685-689, 2012

17. Kämpjärvi K, Kim NH, Keskitalo S, Clark AD, von Nandelstadh P, Turunen M,Heikkinen T,Park MJ,Mäkinen N, KivinummiK, et al: Somatic MED12 mutations in prostate cancer and uterine leiomyomas promote tumorigenesis through distinct mechanisms. Prostate 76: 22-31, 2016.

18. Kämpjärvi K, Mäkinen N, Kilpivaara O, Arola J, Heinonen HR, Böhm J, Abdel-Wahab O, Lehtonen HJ, Pelttari LM, Mehine M, et al: Somatic MED12 mutations in uterine leiomyosarcoma and colorectal cancer. Br J Cancer 107: 1761-1765, 2012.

19. Knuesel MT, Meyer KD, Bernecky C and Taatjes DJ: The human CDK8 subcomplex is a molecular switch that controls Mediator coactivator function. Genes Dev 23: 439-451, 2009.

20. Mittal P, Shin YH, Yatsenko SA, Castro CA, Surti U and Rajkovic A: Med12 gain-of-function mutation causes leiomyomas and genomic instability. J Clin Invest 125: 3280-3244, 2015.

21. Yan H, Parsons DW, Jin G, McLendon R, Rasheed BA, Yuan W, Kos I, Batinic-Haberle I, Jones S, Riggins GJ, et al: IDH1 and IDH2 mutations in gliomas. N Engl J Med 360: 765-773, 2009.

22. Zou Y, Zeng Y, Zhang DF, Zou SH, Cheng YF and Yao YG: IDH1 and IDH2 mutations are frequent in Chinese patients with acute myeloid leukemia but rare in other types of hematological disorders. Biochem Biophys Res Commun 402: 378-383, 2010.

23. Van Raamsdonk CD, Griewank KG, Crosby MB, Garrido MC, Vemula S, Wiesner T, Obenauf AC, Wackernagel W, Green G, Bouvier N, et al: Mutations in GNA11 in uveal melanoma. N Engl J Med 363: 2191-2193, 2010.

24. Clark K, Karsch-Mizrachi I, Lipman DJ, Ostell J and Sayers EW: GenBank. Nucleic Acids Res 44: D67-D72, 2016. 
25. Xu X, Liu J, Zeng X, Lu F, Chen A and Huo X: Elevated serum polybrominated diphenyl ethers and alteration of thyroid hormones in children from Guiyu, China. PLoS One 9: e113699, 2014.

26. Wang W, Liu M, Wang J, Tian R, Dong J, Liu Q, Zhao X and Wang Y: Analysis of the discriminative methods for diagnosis of benign and malignant solitary pulmonary nodules based on serum markers. Oncol Res Treat 37: 740-746, 2014.

27. Henare SJ, Kikuchi M, Talbot RT and Cockrem JF: Changes in plasma gonadotrophins, $17 \beta$-oestradiol, progesterone, prolactin, thyroxine and triiodothyronine concentrations in female Japanese quail (Coturnix coturnix japonica) of a heavy body weight line during photo-induced ovarian growth and regression. Br Poult Sci 53: 520-530, 2012.

28. Matsubara A, Sekine S, Yoshida M, Yoshida A, Taniguchi H, Kushima R, Tsuda H and Kanai Y: Prevalence of MED12 mutations in uterine and extrauterine smooth muscle tumours. Histopathology 62: 657-661, 2013.

29. Osinovskaya NS, Malysheva OV, Shved NY, Ivashchenko TE, Sultanov IY, Efimova OA, Yarmolinskaya MI, Bezhenar VF and Baranov VS: Frequency and spectrum of MED12 exon 2 mutations in multiple versus solitary uterine leiomyomas from Russian patients. Int J Gynecol Pathol 35: 509-515, 2016.

30. Shahbazi S, Fatahi N and Amini-Moghaddam S: Somatic mutational analysis of MED12 exon 2 in uterine leiomyomas of Iranian women. Am J Cancer Res 5: 2441-2446, 2015.

31. Sadeghi S, Khorrami M, Amin-Beidokhti M, Abbasi M, Kamalian Z, Irani S, Omrani M, Azmoodeh O and Mirfakhraie R: The study of MED12 gene mutations in uterine leiomyomas from Iranian patients. Tumour Biol 37: 1567-1571, 2016.

32. Mehine M, Heinonen HR, Sarvilinna N, Pitkänen E, Mäkinen N, Katainen R, Tuupanen S, Bützow R, Sjöberg J and Aaltonen LA: Clonally related uterine leiomyomas are common and display branched tumor evolution. Hum Mol Genet 24: 4407-4416, 2015.
33. Wang H, Ye J, Qian H, Zhou R, Jiang J and Ye L: High-resolution melting analysis of MED12 mutations in uterine leiomyomas in Chinese patients. Genet Test Mol Biomarkers 19: 162-166, 2015.

34. Halder SK, Laknaur A, Miller J, Layman LC, Diamond M and Al-Hendy A: Novel MED12 gene somatic mutations in women from the Southern United States with symptomatic uterine fibroids. Mol Genet Genomics 290: 505-511, 2015.

35. Zhang Q, Ubago J, Li L, Guo H, Liu Y, Qiang W, Kim JJ, Kong B and Wei JJ: Molecular analyses of 6 different types of uterine smooth muscle tumors: Emphasis in atypical leiomyoma. Cancer 120: 3165-3177, 2014.

36. Bertsch E, Qiang W, Zhang Q, Espona-Fiedler M, Druschitz S, Liu Y, Mittal K, Kong B, Kurita T and Wei JJ: MED12 and HMGA2 mutations: Two independent genetic events in uterine leiomyoma and leiomyosarcoma. Mod Pathol 27: 1144-1153, 2014.

37. Makinen N, Vahteristo P, Kämpjärvi K, Arola J, Bützow R and Aaltonen LA: MED12 exon 2 mutations in histopathological uterine leiomyoma variants. Eur J Hum Genet 21: 1300-1303, 2013.

38. Ye J, Wang H, Chen YB, Yuan DL, Zhang LX and Liu L: MED12 mutation in patients with hysteromyoma. Oncol Lett 9: 2771-2774, 2015.

39. Schwetye KE, Pfeifer JD and Duncavage EJ: MED12 exon 2 mutations in uterine and extrauterine smooth muscle tumors. Hum Pathol 45: 65-70, 2014.

40. McGuire MM, Yatsenko A, Hoffner L, Jones M, Surti U and Rajkovic A: Whole exome sequencing in a random sample of North American women with leiomyomas identifies MED12 mutations in majority of uterine leiomyomas. PLoS One 7: e33251, 2012. 